\title{
Radiation hard diamond sensors for future tracking applications
}

\author{
W. Adam ${ }^{\mathrm{a}}$, W. de Boer ${ }^{\mathrm{b}}$, E. Borchi ${ }^{\mathrm{c}}$, M. Bruzzi ${ }^{\mathrm{c}}$, C. Colledani ${ }^{\mathrm{d}}$, P. D'Angelo ${ }^{\mathrm{e}}$, V. Dabrowski ${ }^{\mathrm{f}}$,
} W. Dulinski ${ }^{\mathrm{d}}$, B. van Eijk ${ }^{\mathrm{g}}$, V. Eremin ${ }^{\mathrm{h}}$, F. Fizzotti ${ }^{\mathrm{i}}$, H. Frais-Kölbl ${ }^{\mathrm{j}}$, C. Furetta ${ }^{\mathrm{e}}$, K.K. Gan ${ }^{\mathrm{k}}$, A. Gorisek ${ }^{1}$, E. Griesmayer ${ }^{j}$, E. Grigoriev ${ }^{b}$, F. Hartjes ${ }^{\mathrm{g}}$, J. Hrubec ${ }^{\mathrm{a}}$, F. Huegging ${ }^{\mathrm{m}}$, H. Kagan ${ }^{\text {k,* }}$, J. Kaplon', R. Kass ${ }^{\mathrm{k}}$, K.T. Knöpfle ${ }^{\mathrm{n}}$, M. Krammer ${ }^{\mathrm{a}}$, W. Lange ${ }^{\mathrm{o}}$, A. Logiudice,

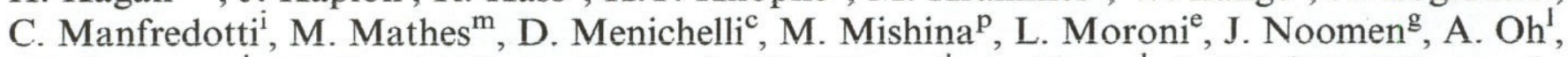
H. Pernegger ${ }^{1}$, M. Pernicka ${ }^{a}$, R. Potenza ${ }^{\mathrm{q}}$, J.L. Riester ${ }^{\mathrm{d}}$, A. Rudge ${ }^{1}$, S. Sala ${ }^{\mathrm{e}}$, S. Schnetzer ${ }^{\mathrm{r}}$, S. Sciortino ${ }^{\text {c }}$, R. Stone ${ }^{r}$, C. Sutera ${ }^{q}$, W. Trischuk ${ }^{\text {s }}$, J.J. Velthuis ${ }^{m}$, B. Vincenzo ${ }^{q}$, P. Weilhammer ${ }^{\mathrm{t}}$, J. Weingarten ${ }^{\mathrm{m}}$, N. Wermes ${ }^{\mathrm{m}}, \mathrm{W}$. Zeuner ${ }^{\mathrm{u}}$

\author{
${ }^{2} H E P H Y$, Vienna, Austria \\ ${ }^{\mathrm{b}}$ Universität Karlsruhe, Karlsruhe, Germany \\ ${ }^{\mathrm{C}}$ University of Florence, Florence, Italy \\ ${ }^{\mathrm{d}}$ LEPSI, IN2P3/CNRS-ULP, Strasbourg, France \\ ${ }^{\mathrm{e} I N F N}$, Milano, Italy \\ ${ }^{\mathrm{f}} U M M$, Cracow, Poland \\ ${ }^{\mathrm{g}} \mathrm{NIKHEF}$, Amsterdam, Netherlands \\ ${ }^{\mathrm{h}}$ Institute for Semiconductor Studies, St. Petersburg, Russia \\ iUniversity of Torino, Torino, Italy \\ ${ }^{\mathrm{j}}$ Fachhocchschule für Wirtschaft und Technik, Wiener Neustadt, Austria \\ 'Ohio State University, Columbus, OH, USA \\ 'CERN, 1211 Geneva, Switzerland \\ ${ }^{\mathrm{m}}$ Universität Bonn, Bonn, Germany

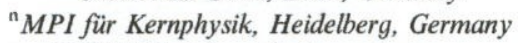 \\ ${ }^{\circ}$ DESY-Zeuthen, Zeuthen, Germany \\ ${ }^{\mathrm{P}}$ FNAL, Batavia, IL, USA \\ ${ }^{\mathrm{q}}$ University of Roma, Italy \\ ${ }^{r}$ Rutgers University, Piscataway, NJ, USA \\ ${ }^{5}$ Department of Physics, University of Toronto, Toronto, Canada \\ 'INFN and University of Perugia, Perugia, Italy \\ "II.Inst. für Exp. Physik, Hamburg, Germany
}

\begin{abstract}
Progress in experimental particle physics in the coming decade depends crucially upon the ability to carry out experiments in high-radiation areas. In order to perform these complex and expensive experiments, new radiation hard technologies must be developed. This paper discusses the use of diamond detectors in future tracking applications and their survivability in the highest radiation environments. We present results of devices constructed with the newest polycrystalline and single crystal Chemical Vapor Deposition diamond and their tolerance to radiation.
\end{abstract}

PACS: 29.40.-n; 29.40.Gx; 29.40.Wk; 29.40.Ym

Keywords: CVD diamond; Radiation hardness; Tracking detector

${ }^{\star}$ Corresponding author. Tel.: + 16142922314 ; fax: + 16142928261.

E-mail address: kagan@mps.ohio-state.edu (H. Kagan). 


\section{Introduction}

Detectors and radiation monitors of future experiments will be situated in radiation environments several orders of magnitude harsher than those of any current detector $[1,2]$. At present detectors for tracking close to the interaction region are based on the mature silicon technology which functions very well in relatively low-radiation environments. However, the practical limits on the radiation hardness of silicon still falls short of what is required for many future experiments.

Chemical Vapor Deposition (CVD) diamond has a number of properties which make it an attractive material for detector applications [2]. Its large band-gap (5.5eV) and large displacement energy (42 eV/atom) make it a material that should be inherently radiation tolerant with very low leakage currents. Its small dielectric constant (5.7) make it a material with low detector capacitances and thereby, low-noise performance of the associated front-end electronics. Its large thermal conductivity (between four and five times that of copper at room temperature) make it a material whose devices may be operated without cooling. CVD diamond is being investigated by the CERN RD42 group.' During the last year, we accomplished the following milestones:

- Improved the charge collection distance of polycrystalline CVD (pCVD) material to above $300 \mu \mathrm{m}$ in wafers with $12 \mathrm{~cm}$ diameter;

- Developed pixel devices useful at the LHC by ATLAS;

- Improved the quality and size of single crystal CVD (scCVD) diamond material;

- Tested the radiation hardness of the highest quality pCVD diamond material up to $20 \times 10^{15} \mathrm{p} / \mathrm{cm}^{2}$;

- Developed a diamond-based system for beam monitoring at ATLAS for the LHC.

This paper discusses items 2-4 listed above.

\section{The first ATLAS diamond pixel module}

The production of high-quality pCVD material [3] with charge collection distance larger than $250 \mu \mathrm{m}$ allowed us to develop applications of diamond to high-energy physics experiments. In collaboration with the groups developing front end electronics for ATLAS and the Fraunhofer Institute for Reliability and Microintegration (IZM) [4] for bump-bonding we constructed a range of diamond pixel detectors. Using the procedures developed for ATLAS, tests performed on $1 \mathrm{~cm}^{2}$ diamond pixel detectors based on older diamond samples showed resolutions with a $1 \mathrm{~cm}^{2}$ ATLAS pixel detector of $12 \mu \mathrm{m}$ in the $x$-view (where the pixels have $50 \mu \mathrm{m}$ pitch) and a characteristic 'top-hat' distribution in $y$ where the pixels are $400 \mu \mathrm{m}$ long. The

'CERN/LHCC Reports 97-3, 98-20, 2000-011, 2000-015, 2001-002, 2002-010, 2003-063, 2005-003, 2006-010. efficiency for finding hits in this pixel detector that correspond to tracks reconstructed in the reference telescope was above $98 \%$. With the success of the $1 \mathrm{~cm}^{2}$ pixel detectors and the availability of the new high collection distance diamond we decided to try to produce a full $2 \mathrm{~cm} \times 6 \mathrm{~cm}$ diamond ATLAS pixel module. This past year, using high-quality diamond, we constructed the first full diamond ATLAS pixel module, bump-bonded it to the final ATLAS IBM $0.25 \mu \mathrm{m}$ rad-hard electronics, and tested the assembly at CERN and DESY.

The diamond discussed in this paper was grown in $12 \mathrm{~cm}$ diameter wafers. In Fig. 1, we show the as-grown diamond wafer ready for test with gold contacts placed at one centimeter intervals.

In the normal production of an ATLAS module, a silicon wafer is laser cut after bump-bonding. However, laser cutting a diamond wafer after bump-bonding graphitizes the surface. Thus, we decided to first remove the $2 \mathrm{~cm} \times 6 \mathrm{~cm}$ diamond part from the wafer, perform the photolithography and then install the diamond in a carrier for bump-bonding. In Fig. 2, we show the diamond after photolithography has been performed mounted in the carrier wafer ready for bump-bonding. In Fig. 3, we show a close-up view of the metal pixel pattern on the diamond after the under-bump metal has been applied.

In Fig. 4, we show the final diamond pixel module with 16 pixel integrated circuit readout chips ready for external cables and testing. This module was tested at CERN using the ATLAS telescope for external tracking. During that data taking period the beam was not centered in the ATLAS telescope yielding very poor tracking results. We were able to obtain an image of the beam in the ATLAS diamond pixel module. In Fig. 5, we show the hit map in the diamond pixel module for events which triggered the pixel module. A clear beam image is evident indicating that all 16 chips work and that a substantial fraction of the detector was operating as expected. The missing stripe in

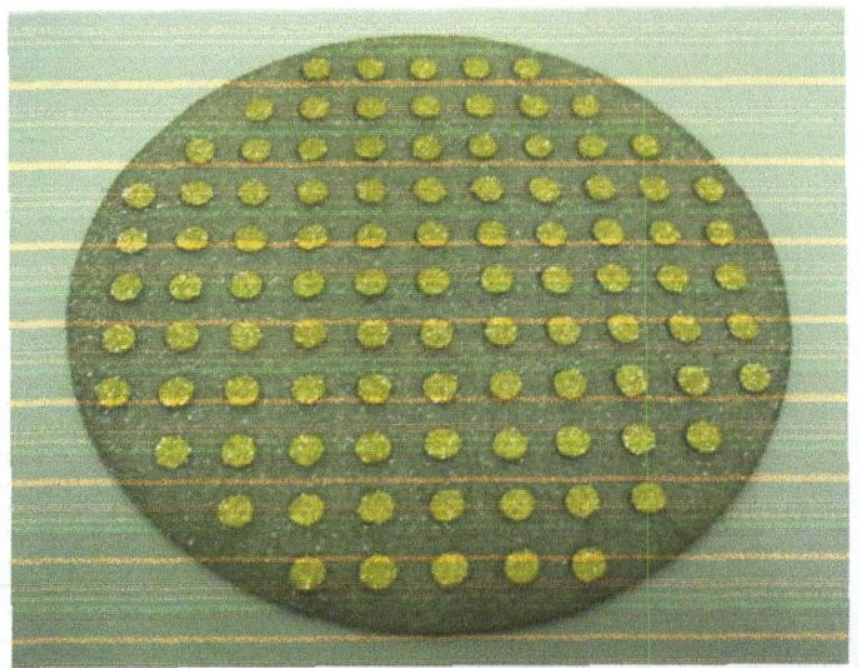

Fig. 1. As grown pCVD wafer ready for test with contacts at $1 \mathrm{~cm}$ intervals. 


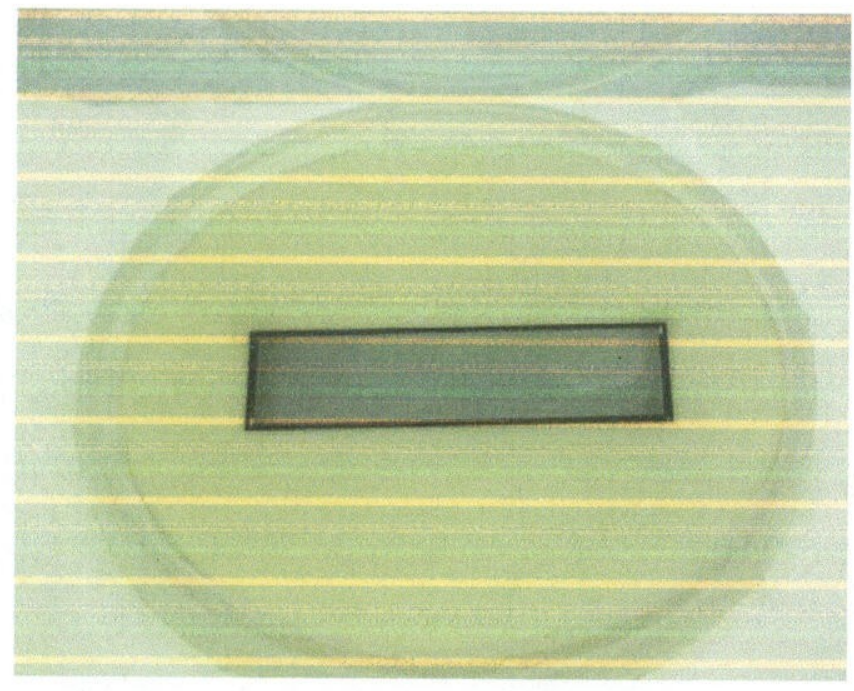

Fig. 2. Photograph of the ATLAS pixel diamond mounted in the carrier ready for bump-bonding.

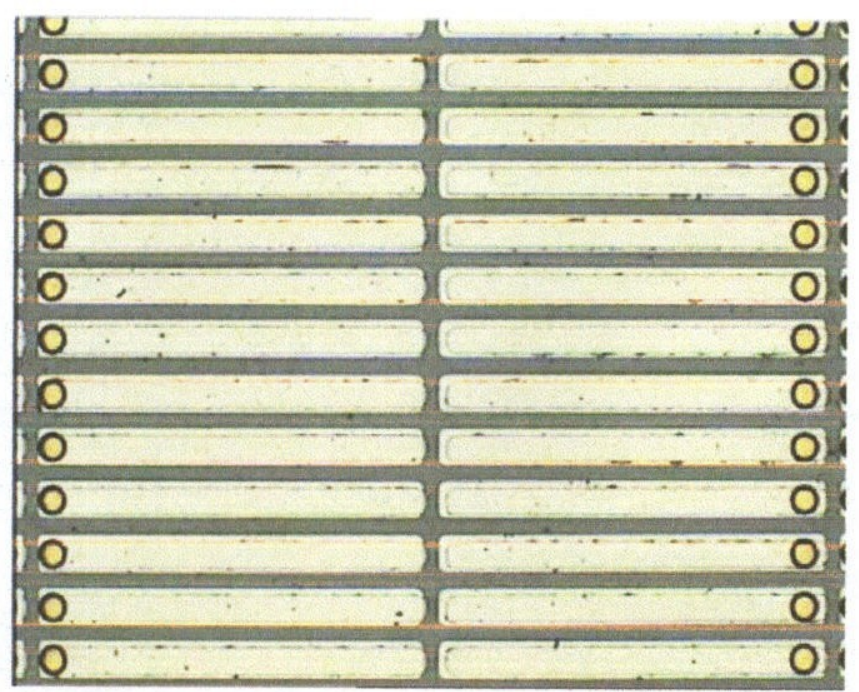

Fig. 3. Zoom view of the pixel pattern after the under-bump metal is deposited.

the CERN data is in the ganged pixel region between the rows of chips where tracking information is required to unravel the pixel ambiguity introduced by the ganging of multiple pixels to a single electronics channel. During this test, the tracking telescope was misaligned and no tracking information was available. In this plot ambiguous hits are not displayed. To continue these tests the diamond module and ATLAS telescope were moved to DESY.

Fig. 6 shows the module noise and threshold obtained in the laboratory before moving to DESY. Fig. 7 shows the tracking results obtained at DESY. Compared to a silicon module the diamond module exhibits smaller noise (136e), can be operated at lower threshold (1450e) and attains a similar efficiency. The spatial resolution observed is $23 \mu \mathrm{m}$ in the $x$-view where the pixels have $50 \mu \mathrm{m}$ pitch and a
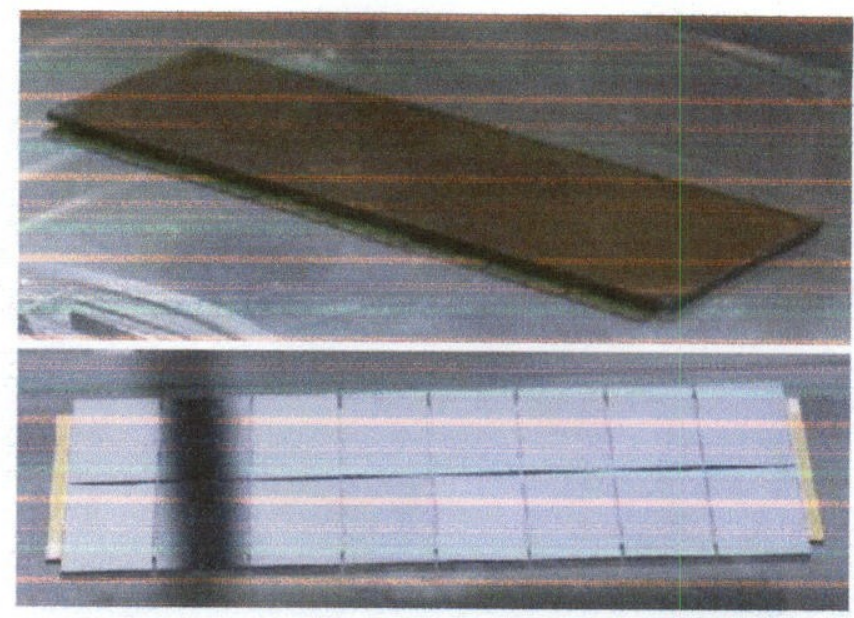

Fig. 4. Photograph of the detector side (upper) and electronics side (lower) of the final ATLAS pixel module.
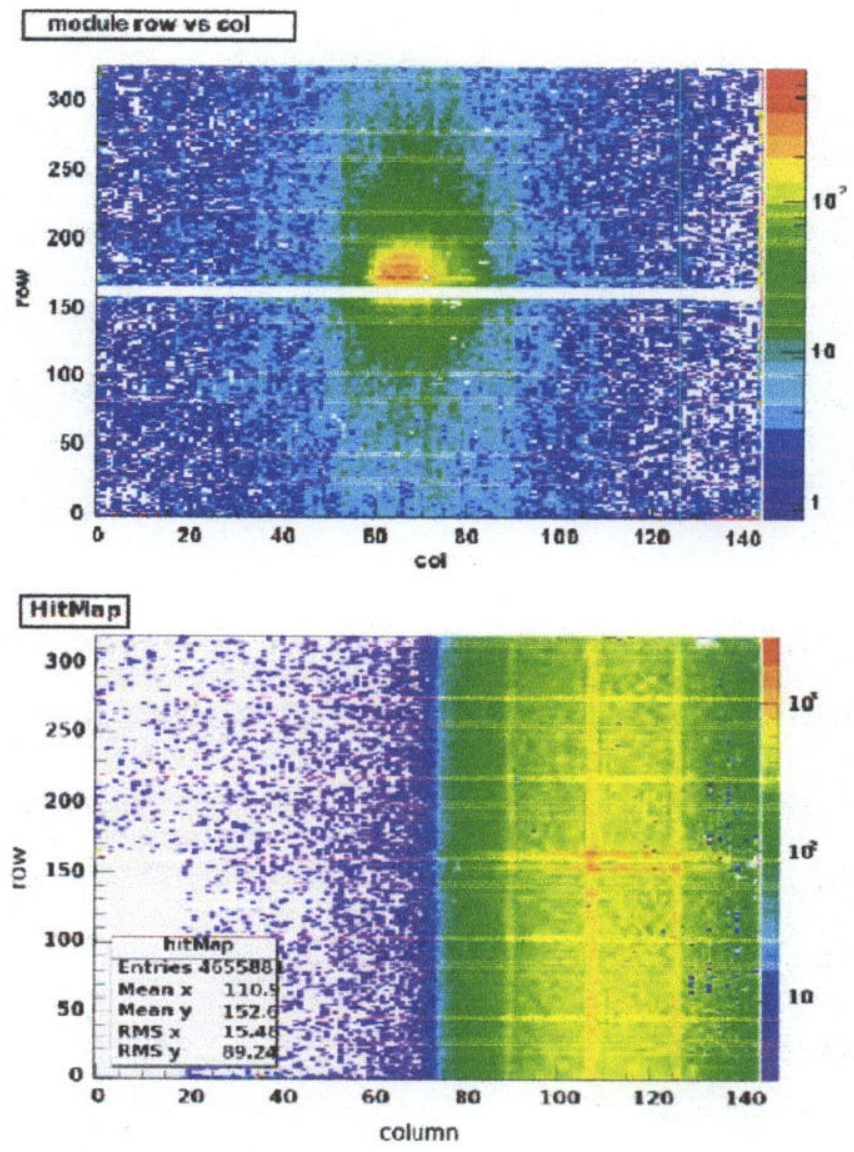

Fig. 5. ATLAS pixel module hitmaps in the $180 \mathrm{GeV}$ pion beam at CERN (upper) and the $4-6 \mathrm{GeV}$ electron beam at DESY (lower). At DESY, with telescope tracking, one observes the edge of the scintillator trigger and that the ganged pixel region has been resolved.

characteristic 'top-hat' distribution in $y$ where the pixels are 400 and $600 \mu \mathrm{m}$ long. In the low-energy $4-6 \mathrm{GeV}$ beam at DESY the spatial resolution is dominated by multiple scattering. For example, the silicon detectors in the 


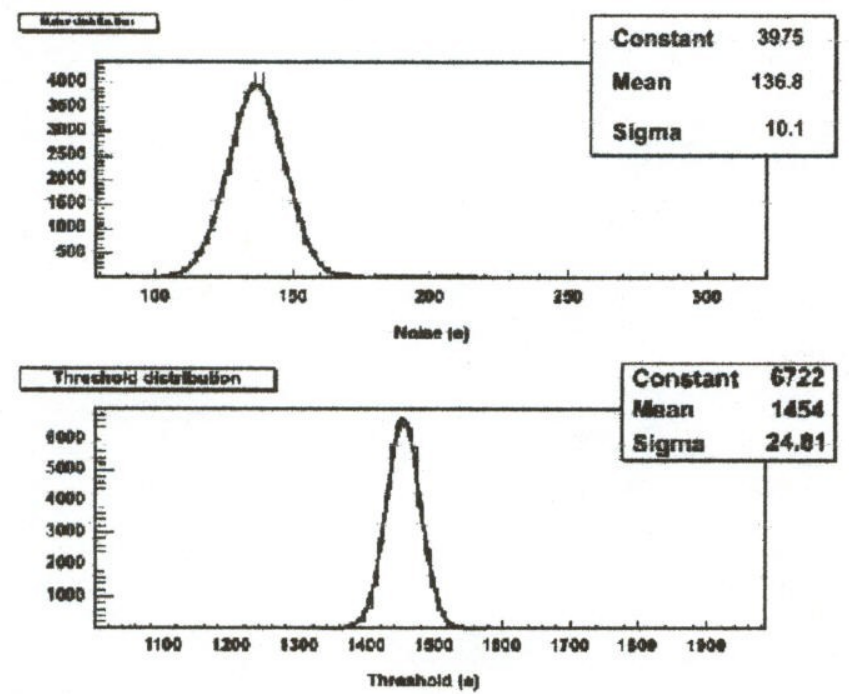

Fig. 6. ATLAS pixeil module results for noise and threshold at DESY.
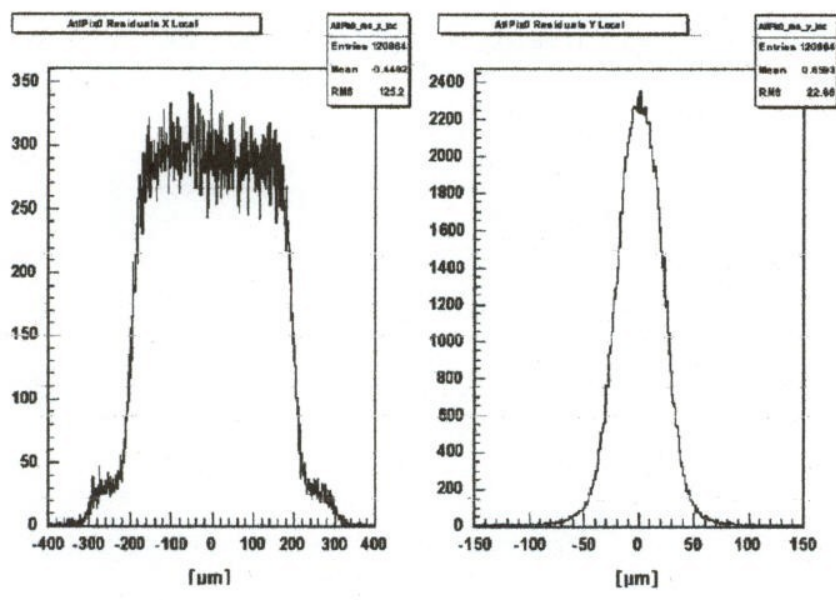

Fig. 7. Pixel module spatial resolution in the test beam at DESY. The contribution from multiple scattering dominates the resolution and has not been unfolded.

telescope have an observed resolution of roughly $7 \mu \mathrm{m}$ per plane at CERN and $37 \mu \mathrm{m}$ per plane at DESY. This generation of pixel detectors, using the newly available larger collection distance diamond, seem suitable for applications at the LHC.

\section{3. scCVD diamond development}

In late fall $2002 \mathrm{RD} 42$ received the first single crystal diamonds grown by a chemical vapor deposition process [5]. The samples were synthesized with a microwave plasma-assisted CVD reactor using a specially prepared $\langle 100\rangle$ oriented single crystal synthetic diamond substrate. These diamonds were typically $0.1-0.2 \mathrm{~cm}^{2}$ in area and $400 \mu \mathrm{m}$ thick. The RD42 group entered into a three year research contract with element six to develop this material. This past year both the thickness and size of the scCVD

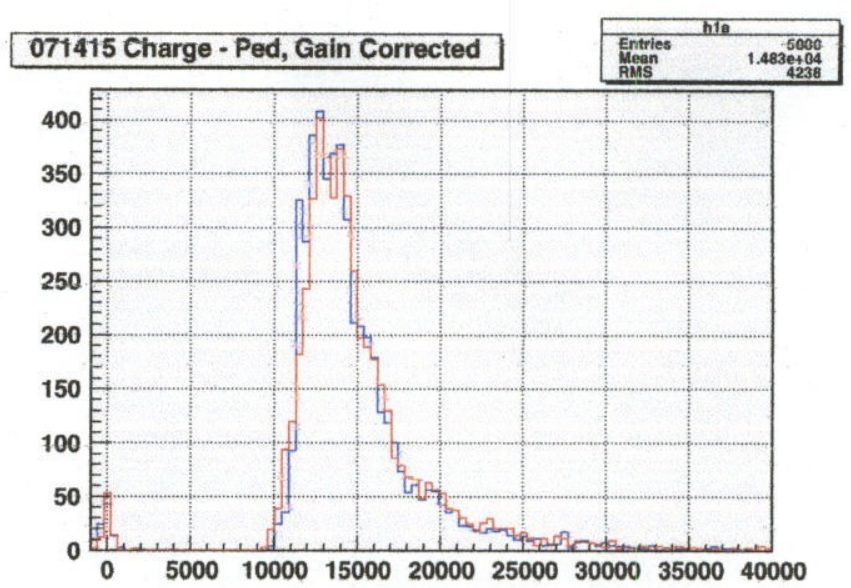

Fig. 8. The pulse height distributions for positive (red) and negative (blue) voltages observed using a $\mathrm{Sr}^{90}$ source from a $450 \mu \mathrm{m}$ thick scCVD diamond.

diamonds was increased. The thickest diamond produced with full charge collection was $770 \mu \mathrm{m}$. The largest area diamond produced was just over $1 \mathrm{~cm}^{2}$.

In Fig. 8, we show the pulse height spectrum observed using a $\mathrm{Sr}^{90}$ from a $450 \mu \mathrm{m}$ thick scCVD diamond pad detector.

We observe a collection distance consistent with full charge collection; most probable charge of $13,400 e$; FWHM of $4000 e$; and more than $10,000 e$ separation between the pedestal and the beginning of the charge distribution. The FWHM/MP for these scCVD diamonds is approximately 0.3 , about one third that of pCVD diamond and about two thirds that of correspondingly thick silicon.

In Fig. 9 we show the most probable charge observed in scCVD diamond using a $\mathrm{Sr}^{90}$ source versus thickness of the material. A clear linear relationship is evident out to thicknesses of $770 \mu \mathrm{m}$.

In order to understand the basic properties of this new material we began a program to measure the carrier lifetime and mobility by observing the transient current pulse when a particle penetrates a diamond and stops near the entrance electrode. In Fig. 10, we show the current pulses created by alpha particles injected on one surface of the scCVD diamond as a function of time [6]. Each trace represents a different setting of bias voltage. The fast rising of the current signal is dominated by the electronic time constant of the amplifier and readout system and remains approximately constant at higher voltages. The falling edge clearly shows the arrival of the charge cloud at the opposite electrode for electrons at all voltage settings and for holes at biases with magnitude above $80 \mathrm{~V}$. The decrease of current signal during the drift for holes and increase in current signal for electrons can be interpreted as the convolution effect of negative effective space charge concentration in the bulk and trapping of charge carriers. The parameters extracted from this data, independently for electrons and holes, include transit time, drift velocity and mobility as a function of electric field, and carrier lifetime 


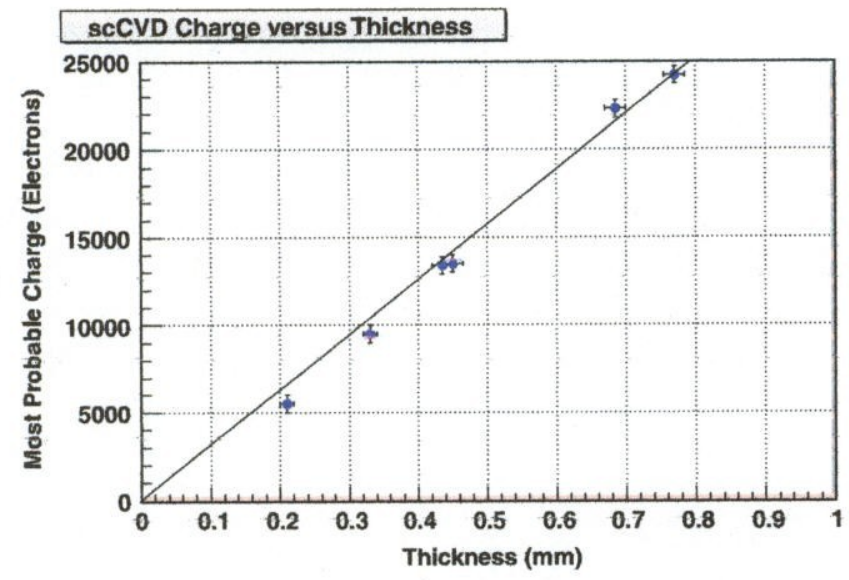

Fig. 9. The most probable pulse height versus thickness for scCVD diamonds.
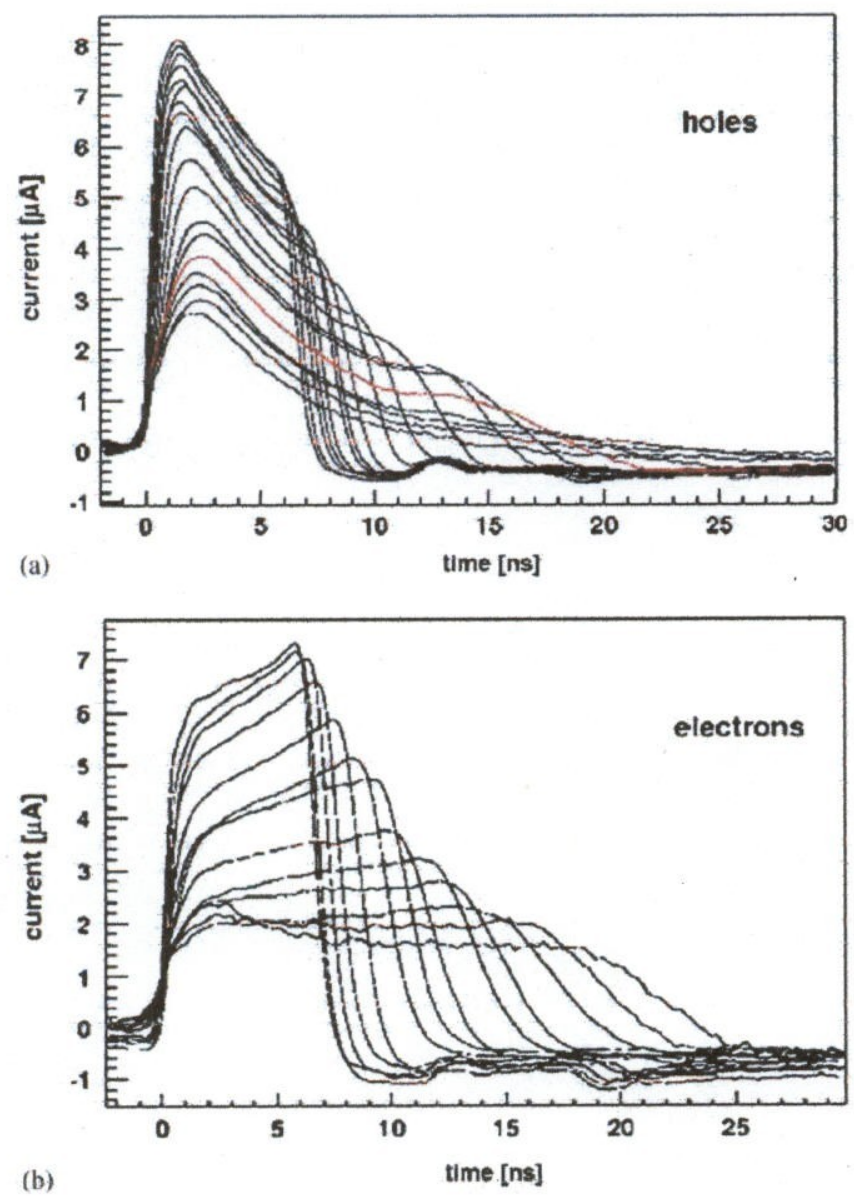

Fig. 10. The observed current pulses created by alpha particles injected on one surface of the SCVD diamond as a function of time. The different curves are for different voltages in the range $-40--375$ volts and $+70-+690$ volts: (a) shows the hole pulses resulting from negative values of applied voltage of $40,50,60,70,90,100,125,150,175,200,225,250$, $275,300325,350$, and $375 \mathrm{~V}$ (from the bottom to the top of the graph); (b) shows the electron pulses resulting from positive values of the applied voltage of $70,85,100,125,150,200,250,300,400,500,600,650$, and $690 \mathrm{~V}$. and space charge. Our first results $[6]$ indicate that the carrier lifetimes (both electrons and holes) in scCVD diamond are of the order of $35 \mathrm{~ns}$ and that the particular diamond we studied has an internal space charge. Results such as this should help in our understanding of the CVD process and in the manufacturing of still better material. Based on our test results, this new material seems extraordinary: scCVD diamond may resolve many if not all of the issues associated with polycrystalline material.

\section{Radiation hardness studies with pCVD diamond trackers}

The results from previous irradiations $\{7,8]$ show that up to $2.2 \times 10^{15} \mathrm{p} / \mathrm{cm}^{2}$ pCVD diamonds lose at most $15 \%$ of the most probable charge and improve their resolution by roughly $40 \%$. In Fig. 11, we show the previous results for the collected charge from a polycrystalline diamond strip detector after irradiation with a fluence of $24 \mathrm{GeV}$ protons of $1 \times 10^{15} \mathrm{p} / \mathrm{cm}^{2}$ and after $2.2 \times 10^{15} \mathrm{p} / \mathrm{cm}^{2}$. While the strip contacts before and after irradiation with fluences of $1 \times 10^{15} \mathrm{p} / \mathrm{cm}^{2}$ were unchanged the contacts were replaced after a fluence of $2.2 \times 10^{15} \mathrm{p} / \mathrm{cm}^{2}$ and then characterized in the test beam. This step was necessary since the wire bond pads were only usable twice. At $1 \times 10^{15} \mathrm{p} / \mathrm{cm}^{2}$ we observe that the shape of the signal-to-noise distribution is narrower than before irradiation and entries in the tail of the distribution appear closer to the most probable signal. At $2.2 \times 10^{15} \mathrm{p} / \mathrm{cm}^{2}$ and after re-metalization we observe essentially the same signal-to-noise distribution (measured with a low-noise VA chip amplifier) as at $1 \times 10^{15} \mathrm{p} / \mathrm{cm}^{2}$ indicating that very little further damage occurred to the diamond bulk. The most probable signal-to-noise was 41 before irradiation and 35 at $1 \times 10^{15} \mathrm{p} / \mathrm{cm}^{2}$ and also at $2.2 \times 10^{15} \mathrm{p} / \mathrm{cm}^{2}$. We find a reduction of maximum $15 \%$ in

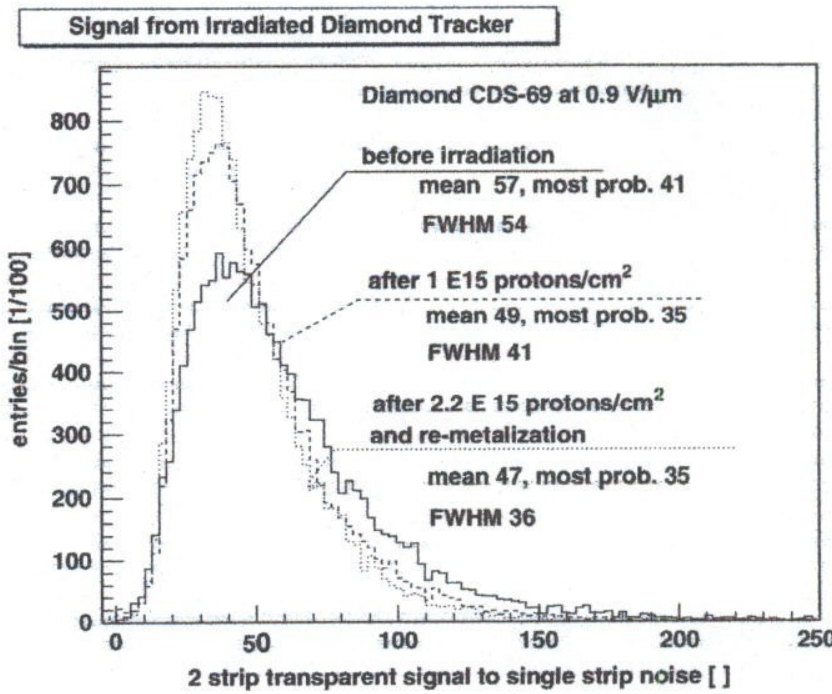

Fig. 11. Transparent 2-strip charge signal-to-noise distributions before (solid line), after proton irradiations with $1 \times 10^{15} \mathrm{p} / \mathrm{cm}^{2}$ (dashed line) and after $2.2 \times 10^{15} \mathrm{p} / \mathrm{cm}^{2}$ (dotted line). 


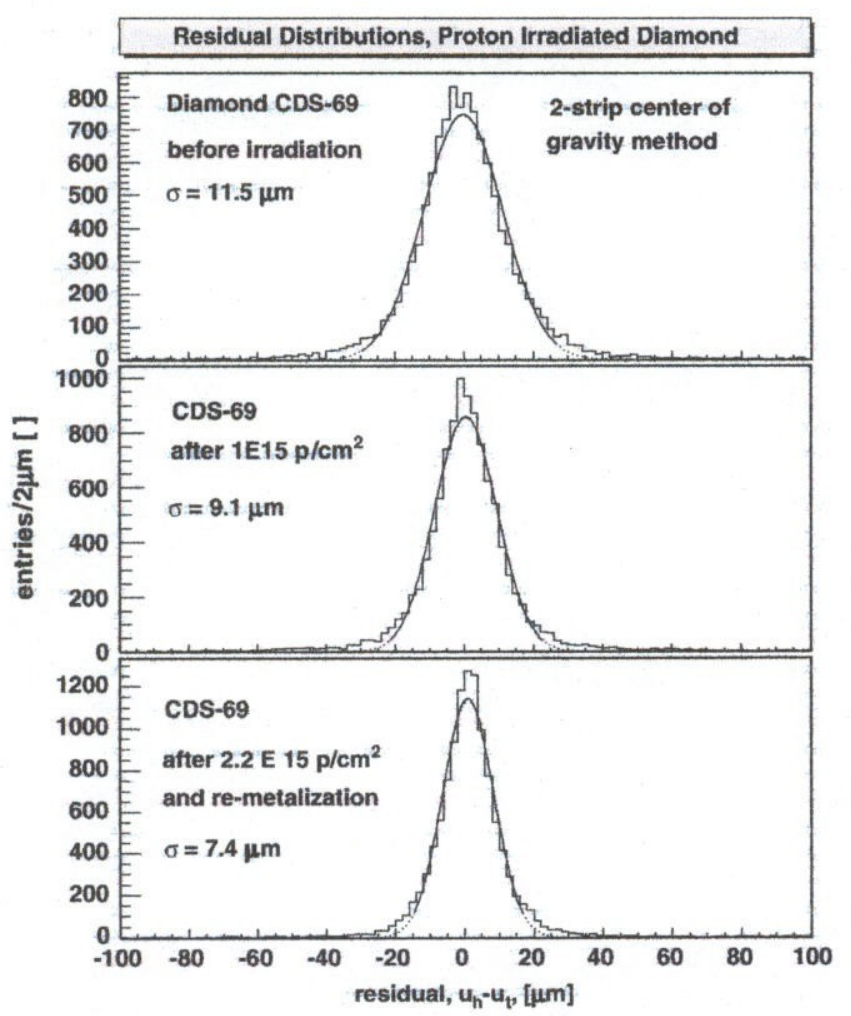

Fig. 12. Residual distributions before and after proton irradiation.

the most probable signal-to-noise after irradiation with $2.2 \times 10^{15} \mathrm{p} / \mathrm{cm}^{2}$. The noise was measured to remain constant at each beam test. Since the beam test with the detector irradiated with a fluence of $2.2 \times 10^{15} \mathrm{p} / \mathrm{cm}^{2}$ used new contacts the observed decrease of $15 \%$ is attributed to damage in the diamond bulk.

Fig. 12 shows residual distributions before irradiation, after $1 \times 10^{15} \mathrm{p} / \mathrm{cm}^{2}$ and after $2.2 \times 10^{15} \mathrm{p} / \mathrm{cm}^{2}$. We observe that the spatial resolution improves from (11.5 \pm $0.3) \mu \mathrm{m}$ before irradiation to $(9.1 \pm 0.3) \mu \mathrm{m}$ at $1 \times$ $10^{15} \mathrm{p} / \mathrm{cm}^{2}$ and to $(7.4 \pm 0.2) \mu \mathrm{m}$ at $2.2 \times 10^{15} \mathrm{p} / \mathrm{cm}^{2}$. At present, the explanation for this effect is that the irradiated material is more uniform in the sense that the probability of large landau fluctuations has been reduced by the irradiation. The spatial resolution of nearly $7 \mu \mathrm{m}$ with a detector of $50 \mu \mathrm{m}$ strip pitch is comparable to results obtained with silicon detectors.

The RD42 group has now extended these irradiations to fluences up to $20 \times 10^{15} \mathrm{p} / \mathrm{cm}^{2}$ for pCVD material. This

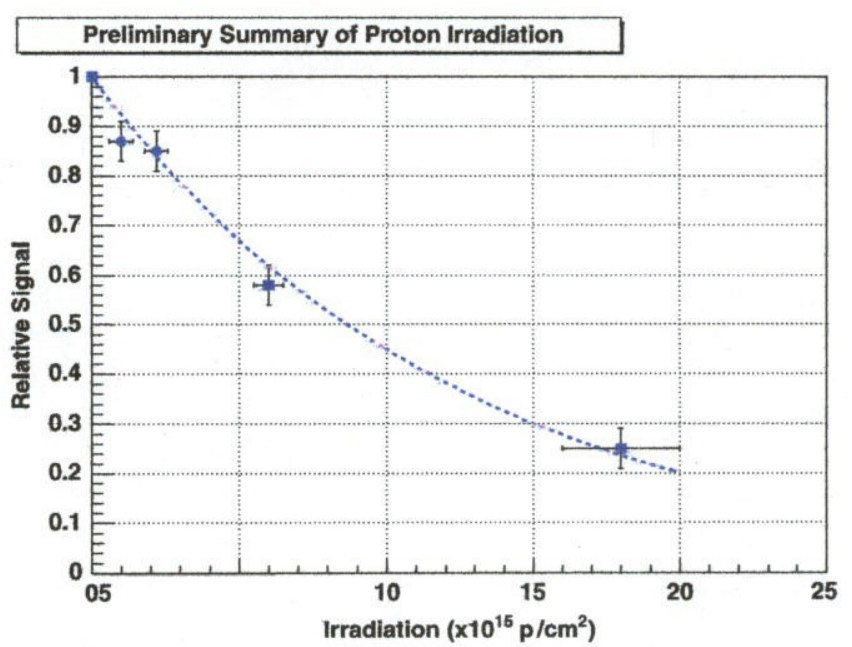

Fig. 13. Summary of proton irradiation results for pCVD material up to a fluence of $20 \times 10^{15} \mathrm{p} / \mathrm{cm}^{2}$ (filled data points). The blue curve is an exponential with exponent $-0.08 \times$ fluence indicating a radiation lifetime for this material of $12.5 \times 10^{15} \mathrm{p} / \mathrm{cm}^{2}$.

figure corresponds to a dose of roughly $500 \mathrm{Mrad}$. At this fluence the pCVD diamond retains $25 \%$ of its original pulse height when operated at an electric field of $1 \mathrm{~V} / \mu \mathrm{m}$. In Fig. 13, we show a summary of the proton irradiation results described above. We find that all of the irradiations fall along an exponential curve. At an electric field of $1 \mathrm{~V} / \mu \mathrm{m}$ the diamond signal is down by $1 / e$ at $12.5 \times 10^{15} \mathrm{p} / \mathrm{cm}^{2}$.

\section{Conclusion}

Indications are that this latest diamond material processed in this simple manner is suitable for applications in very high-radiation environments such as the LHC.

\section{References}

[1] First Workshop on Radiation Hard Semiconductor Devices for Very High Luminosity Colliders, CERN, 28-30 November 2001.

[2] M. Franklin, et al., Nuct. Instr. and Meth. A 315 (1992) 39.

[3] Element Six Ltd., King's Ride Park, Ascot, Berkshire SL5 9BP UK.

[4] Fraunhofer Institut Zuverlassigkeit Mikrointegration, Gustav-MeyerAllee 25, D-13355, Berlin, Germany.

[5] J. Isberg, et al., Science 297 (2002) 1670.

[6] H. Pernegger, et al., J. Appl. Phys. 97 (2005) 073704.

[7] D. Meier, et al., Nucl. Instr. and Meth. A 426 (1999) 173.

[8] W. Adam, et al., Nucl. Instr. and Meth. A 447 (2000) 244. 